\title{
HELICON PLASMA INJECTOR AND ION CYCLOTRON ACCELERATION DEVELOPMENT IN THE VASIMR EXPERIMENT
}

\author{
Jared P. Squire*, Franklin R. Chang Díaz ${ }^{\dagger}$, Verlin T. Jacobson ${ }^{\ddagger}$, and Greg E. McCaskill ${ }^{\S}$ \\ Advanced Space Propulsion Laboratory, JSC / NASA, Houston, TX \\ Roger D. Bengtson ${ }^{9}$ \\ The University of Texas at Austin, Austin, TX \\ Richard H. Goulding ${ }^{\#}$ \\ Oak Ridge National Laboratory, Oak Ridge, TN
}

\begin{abstract}
In the Variable Specific Impulse Magnetoplasma Rocket (VASIMR) radio frequency (rf) waves both produce the plasma and then accelerate the ions. The plasma production is done by action of helicon waves. These waves are circular polarized waves in the direction of the electron gyromotion. The ion acceleration is performed by ion cyclotron resonant frequency (ICRF) acceleration. The Advanced Space Propulsion Laboratory (ASPL) is actively developing efficient helicon plasma production and ICRF acceleration. The VASIMR experimental device at the ASPL is called VX-10. It is configured to demonstrate the plasma production and acceleration at the $10 \mathrm{~kW}$ level to support a space flight demonstration design. The VX-10 consists of three electromagnets integrated into a vacuum chamber that produce magnetic fields up to 0.5 Tesla. Magnetic field shaping is achieved by independent magnet current control and placement of the magnets. We have generated both helium and hydrogen high density $\left(>10^{18} \mathrm{~m}^{-3}\right)$ discharges with the helicon source. ICRF experiments are underway. This paper describes the VX-10 device, presents recent results and discusses future plans.
\end{abstract}

\footnotetext{
*Senior Research Scientist, Muñiz Engineering, Inc. ${ }^{\dagger}$ ASPL Director, NASA Astronaut.

${ }^{\ddagger}$ Research Staff, Muñiz Engineering, Inc.

${ }^{\S}$ Electrical Engineer Staff, Muñiz Engineering, Inc.

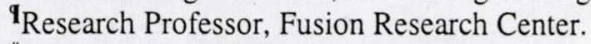

\#Research Staff, Fusion Energy Division.
}

Copyright (C) 2000 by the American Institute of Aeronautics and Astronautics, Inc. No copyright is asserted in the United States under Title 17, U.S. Code. The U.S. Government has a royalty-free license to exercise all rights under the copyright claimed herein for Government Purposes. All other rights are reserved by the copyright owner.

\section{INTRODUCTION}

High power density plasma propulsion would vastly improve human exploration of our solar system. The fusion energy research community has long known that magnetized plasma systems enable high energy density discharges, and that the magnetic field insulates the material wall from the hot plasma. Radio frequency (rf) waves have proven to be efficient at plasma production in helicon ${ }^{1}$ devices, where a right-hand circularly polarized wave is launched into the plasma. Also in fusion research, ion cyclotron resonant heating (ICRH) ${ }^{2}$ has been very successful at producing energetic ions without having electrodes or grids in contact with the intense plasma. The same concept of $\mathrm{rf}$ production and heating of magnetized plasmas can be used for high performance propulsion.

Efficient helicon plasma production and injection into a strong magnetic field is crucial for the performance of the Variable Specific Impulse Magnetoplasma Rocket (VASIMR) concept. ${ }^{3,}{ }^{4}$ The subsequent ICRF acceleration of the ions via $\mathrm{rf}$ driven waves is necessary to produce significant thrust. The experiment in the Advanced Space Propulsion Laboratory (ASPL) at the NASA Johnson Space Center is presently focused on research in these two areas. The experimental device in the ASPL is called VX-10. It is configured to operate in the $10 \mathrm{~kW}$ power range to support the design of an early space flight demonstration of the VASIMR concept. $^{5}$ This paper reports on the status of the helicon plasma source development and ICRF acceleration research.

\section{THE VX-10 EXPERIMENT}

The VX-10 experiment is quickly evolving to a configuration that demonstrates the plasma production and ion acceleration components of the VASIMR concept. The VX-10 will soon operate at a total power 

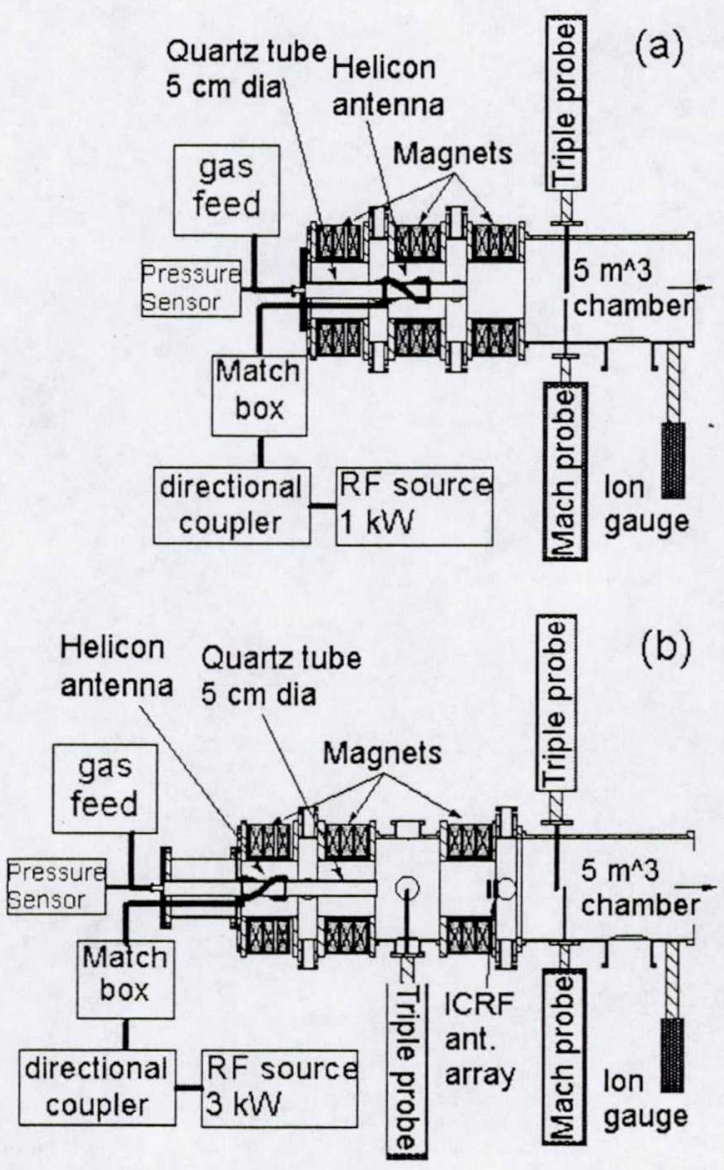

FIG. 1. Experiment configurations. (a) Helium helicon study configuration. (b) Low field helicon configuration with magnetic choke for hydrogen experiments.

level of $10 \mathrm{~kW}$ and targets a specific impulse of 5,000 to 10,000 seconds. Figure 1 shows two main configurations in the recent experimental evolution. The primary focus of the work so far has been helicon plasma production with helium and hydrogen. The earlier configuration (Fig. 1a) was designed to produce an almost uniform magnetic field for just helicon study. The later configuration incorporates what we call a low field helicon section and magnetic choke. This configuration also has shifted the helicon antenna location and moved the gas injection plate to a much lower magnetic field location. The ICRF antenna is installed near the magnetic choke. Figure 2 shows the VX-10 in operation.

In the present configuration (Fig. 1b), neutral gas (hydrogen or helium in the present data) is injected at less than or about $1 \mathrm{mg} / \mathrm{s}$ into an axisymmetric magnetic field that is shaped in strength along the axis.
The injected neutral gas is contained in a quartz tube (5 $\mathrm{cm}$ in diameter) until it is ionized by an $\mathrm{rf}$ driven helicon wave in a magnetic field on the order of 0.1 Tesla. The plasma then flows near the sound speed, about $10^{4} \mathrm{~m} / \mathrm{s}$, into a high magnetic field, about 0.2 Tesla for hydrogen, region where ICRF acceleration takes place. The accelerated ions $(\sim 100 \mathrm{eV})$ move out in an expanding magnetic nozzle where perpendicular velocity is converted to axial velocity.

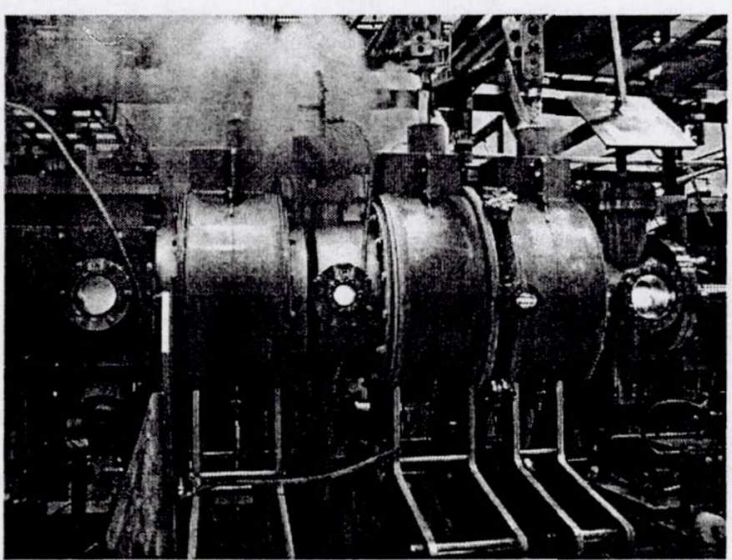

FIG. 2. The VX-10 device running.

The magnetic field in the experiment is produced using three independently driven liquid nitrogen cooled copper coil electromagnets capable of a maximum field of 0.3 Tesla for 10 minutes. A one Tesla capability for short pulses, 30 seconds, is being implemented. Light weight high temperature superconducting magnets are being developed to replace the low cost copper magnets. ${ }^{6}$ The VX-10 will soon start evolving to a superconducting magnet system.

A precision mass flow controller (MFC) measures and regulates gas flow into the system. The MFC range is 10 - 1000 standard cubic centimeters per minute $(\mathrm{sccm})$. This corresponds to maximum mass flow of about $1.5 \mathrm{mg} / \mathrm{second}$ for hydrogen. The MFC also has $100 \mathrm{~ms}$ time response for gas pulsing experiments. A baratron measures the pressure in the gas inlet. A magnetically shielded ion gauge measures the neutral pressure in the vacuum chamber at the plasma exhaust region. The plasma exhausts into a $5 \mathrm{~m}^{3}$ vacuum chamber, which is pumped at 5000 liters/second. Components for a 50,000 liter/second pump upgrade have been acquired and installation is being planned.

In the helicon section, a water-cooled half-turn helical antenna is driven at $7-50 \mathrm{MHz}$ with up to $3 \mathrm{~kW}$ of 


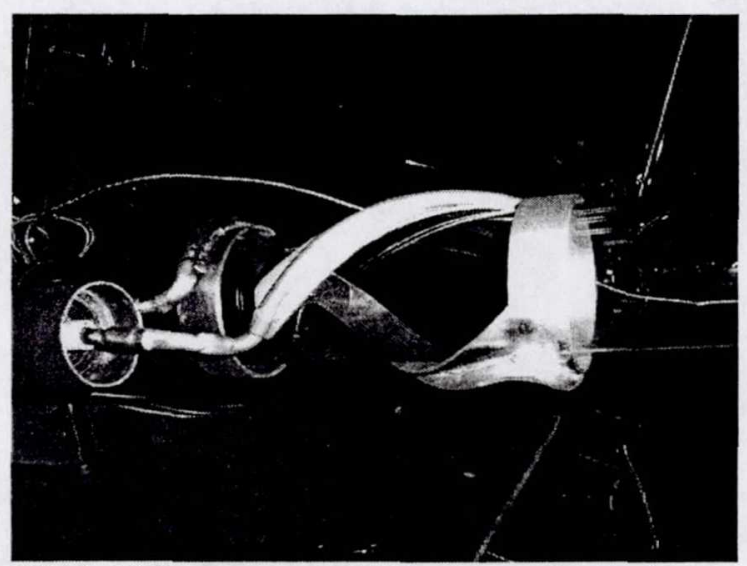

FIG. 3. Helicon antenna with the quartz tube running through it.

power. Figure 3 shows a photograph of an antenna. The $50 \mathrm{Ohm}$ transmission line is matched to the antenna impedance using two vacuum variable capacitors in a simple network. Forward and reflected powers are measured with a directional coupler in the impedance matched section on the transmitter side of the matching network.

A water-cooled phased double half-turn antenna array is installed to launch waves at about $3 \mathrm{MHz}$ for ICRF acceleration of the ions. The antenna array is install just downstream of the magnetic choke and just upstream from the ion resonance. The two antenna rings are separated about $0.03 \mathrm{~m}$ axially and the $\mathrm{rf}$ power feeds are rotated $90^{\circ}$. Figure 4 shows the antenna array being installed.

A $100 \mathrm{~kW}$ rf steady state source is in operation at 3 $\mathrm{MHz}$ to drive the antenna array, although the array is driven only at the $10 \mathrm{~kW}$ power level. The power splitting, phasing, and impedence matching networks have been installed and tested.

\section{DIAGNOSTICS}

Reciprocating Langmuir and Mach probes are the primary plasma diagnostics. The Langmuir probe measures electron density and temperature profiles while the Mach probe measures flow profiles. Together this gives total plasma particle flux. The Langmuir probe has four molybdenum tips that are biased as a triple probe ${ }^{7}$, with an extra tip for measuring electrostatic fluctuations. The Mach probe has two molybdenum tips biased in ion saturation, one upstream and one downstream of a stainless steel separator. ${ }^{8}$

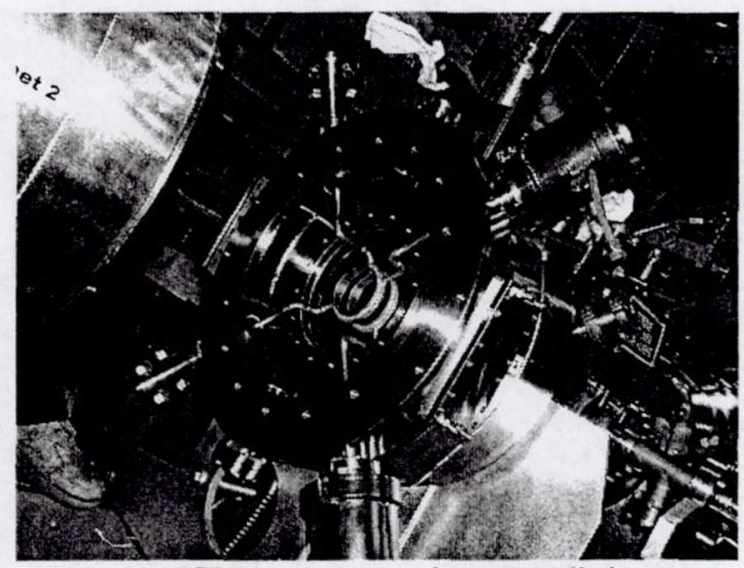

FIG. 4. The ICRF antenna array being installed

Retarding potential analyzer (RPA) diagnostics are being installed to measure the accelerated ions. A 70 $\mathrm{GHz}$ microwave density interferometer will soon be installed in the plasma exhaust region. It will be located about $0.3 \mathrm{~m}$ downstream of the ICRF resonance. There is also a CCD camera installed in the vacuum chamber that has an oblique view of the antennas and discharge.

Accounting for all of the power losses in the system is very important, so we have added several diagnostics to measure these quantities. Water calorimeters measure heat loads on all of the antennas. Thermocouples are used to measure heat loads on components such as the quartz tube. A bolometer has been recently installed to measure the total radiated power. To compliment these measurements, we are installing rf antenna current and voltage diagnostics to determine the plasma loaded power from the antennas. This is an active area of study, so results will be presented in subsequent papers.

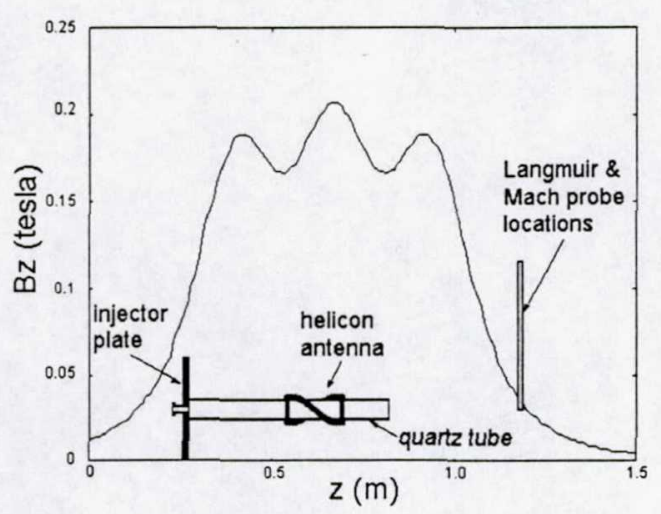

FIG. 5 A typical axial magnetic field profile corresponding to Fig. 1a. The antenna configuration and Langmuir probe locations are shown. 

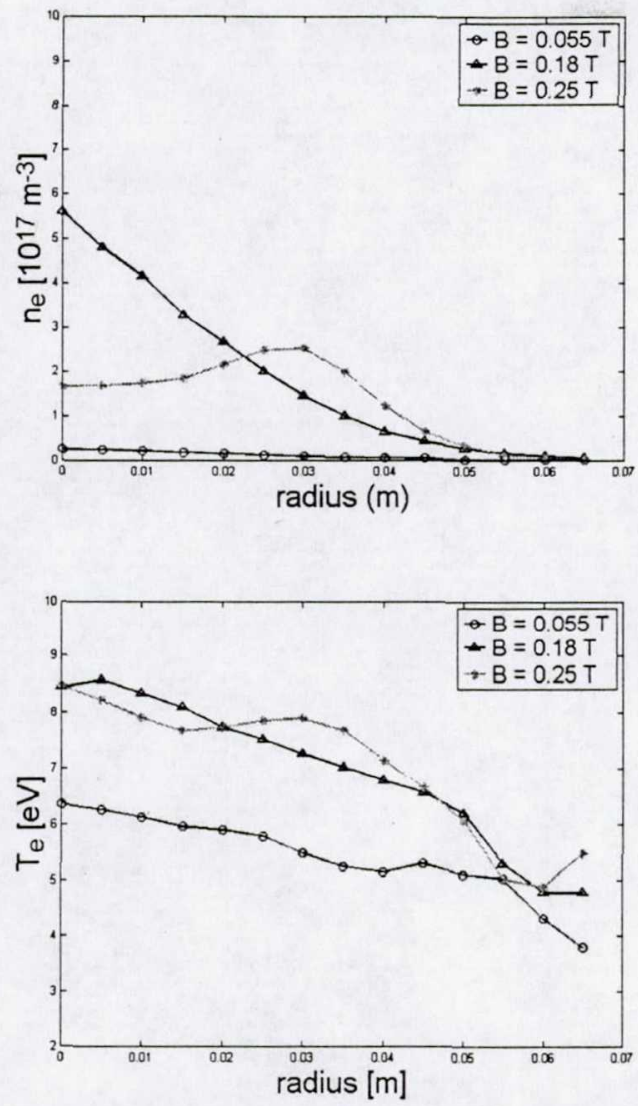

FIG. 6 Radial profiles for $600 \mathrm{~W}$ at $7 \mathrm{MHz}$ with helium. (a) The electron density profile. (b) The electron temperature profile.

\section{HELICON RESULTS}

Configuration variations of the helicon section are being explored to optimize plasma production efficiency. Both hydrogen and helium discharges studies have been performed. Many parameters are varied, such as: rf drive frequency and power; magnetic field shape, strength and polarization; quartz tube length and diameter; antenna structure and location; and gas flow rates.

\section{HELIUM}

Parametric studies presented here start with helium discharges in a fairly uniform magnetic field, and no magnetic choke. A typical magnetic field axial profile is shown in Fig. 5. In this study, the helicon antenna is nearly centered in the three-magnet set, shown in Fig. 1a. The quartz tube containing neutral gas extends through the antenna and a short distance beyond. The three magnets were configured in series and spaced equally.
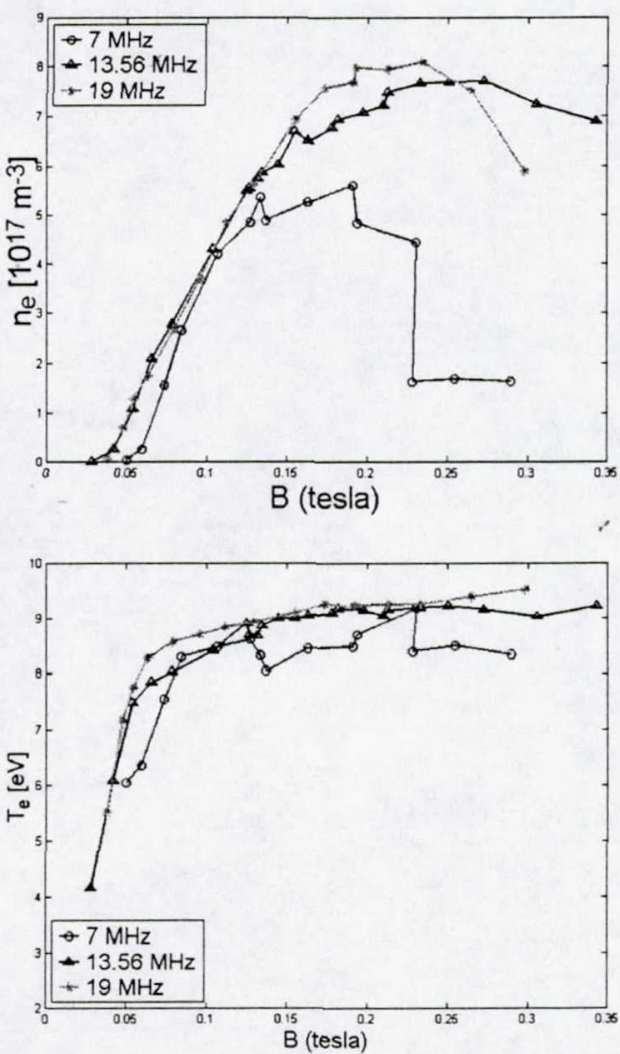

FIG. 7. Magnetic field scan for three rf frequencies. (a) The central electron density. (b) The central electron temperature.

In this configuration, we scanned the gas flow, magnetic field and input power for three rf drive frequencies. Figure 6 shows typical radial profiles of density and temperature for a $7 \mathrm{MHz}$ driven case. (There is some small uncertainty to the plasma center, but the $\mathrm{r}=0$ point on the graph is within $1 \mathrm{~cm}$ of the center.) The density profiles are usually peaked and the temperature profiles are flat. We did see a mode change to a hollow profile at higher magnetic fields with the $7 \mathrm{MHz}$ drive frequency. The $0.18 \mathrm{~T}$ profiles shown in Fig. 6 have a typical shape of the other $\mathrm{rf}$ drive frequency cases.

In Fig. 7 we see the effect of scanning only the magnetic field strength. Power is fixed at $600 \mathrm{~W}$ and gas flow at $200 \mathrm{sccm}\left(9 \times 10^{19} / \mathrm{sec}\right)$ of helium. The plasma density rise starts at or about when the rf frequency equals the lower hybrid frequency $\left(\omega_{L H}\right)$. as defined by the following equation ${ }^{9}$

$$
\frac{1}{\omega_{L H}^{2}}=\frac{1}{\omega_{c e} \omega_{c i}}+\frac{1}{\omega_{p i}^{2}+\omega_{c i}^{2}} .
$$



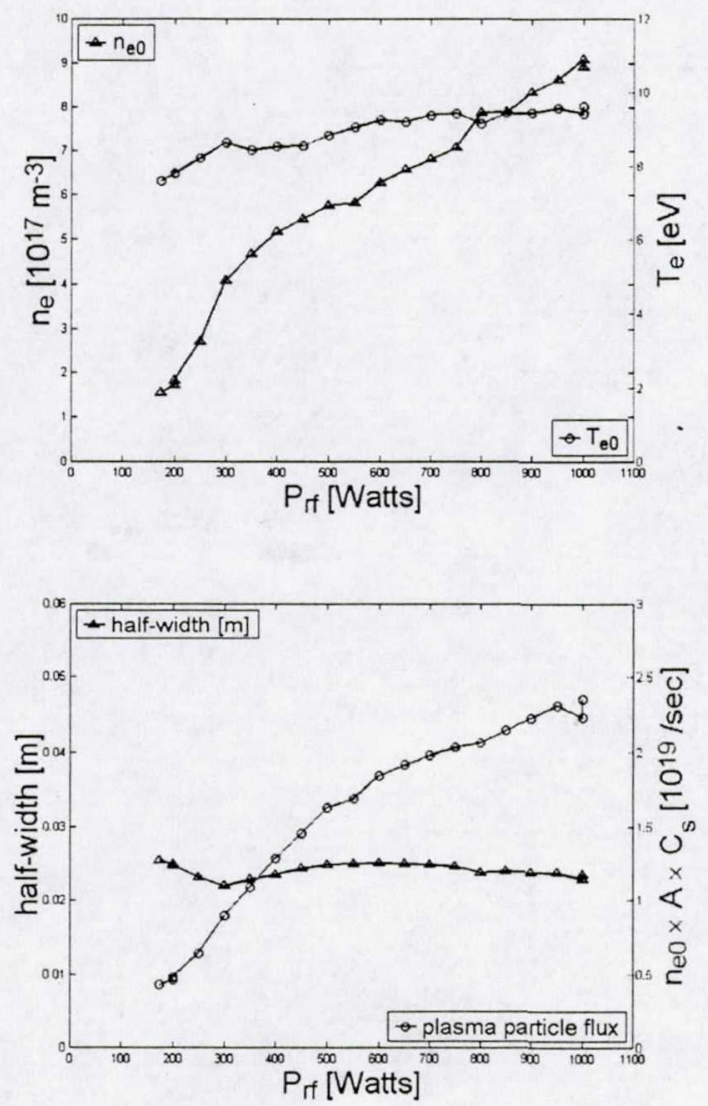

FIG. 8 Power scan at $13.56 \mathrm{MHz}$ with $200 \mathrm{sccm}$ of helium. (a) Central electron density and temperature. (b) Profile half-width and plasma flux estimate.

The density peaks when $\omega_{r f}=0.25 \omega_{L H}$. The density quickly falls off at higher magnetic fields. The electron temperature, shown in Fig. 7b, rises quickly at the first density rise, but then changes very little. The higher frequency generates a slightly higher temperature. These results indicate that higher frequency operation could lead to better plasma production performance.

Figure 8 shows the result of scanning the rf power for a 13.56 MHz drive frequency with $200 \mathrm{sccm}$ mass flow of helium. Below about $150 \mathrm{~W}$, a high density helicon discharge can not be generated, but a very low density discharge is still seen. After the helicon discharge is well started, above $300 \mathrm{~W}$, the density rises almost linearly with the applied power. The electron temperature rises very slowly and stays about $7 \mathrm{eV}$. The profile shape almost stays constant as shown by the half-width plotted in Fig. 8b.
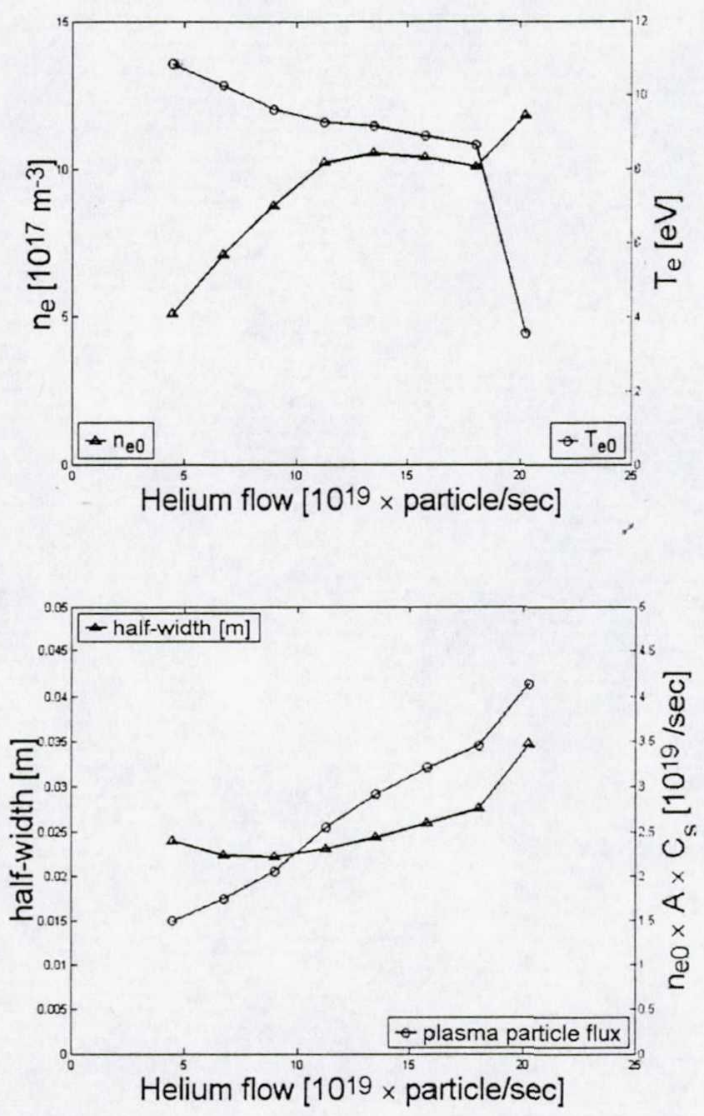

FIG. 9 Injected gas flow scan for $1000 \mathrm{~W}$ at $13.56 \mathrm{MHz}$. (a) Central electron density and temperature. (b) Profile half-width and plasma flux estimate.

Also shown in Fig. 8b is an estimate of the total plasma flux $\left(\Gamma_{\text {plasma }}\right)$ in the exhaust. This is estimated by

$$
\Gamma_{\text {plasma }} \approx n_{e 0} \cdot A \cdot C_{s},
$$

where $n_{e 0}$ is the central electron density, $A$ is the effective cross sectional area of the plasma colume as determined by the Langmuir probe profile, and $C_{s}=\sqrt{T_{e} / m_{i}}$ is the plasma sound speed. This formula assumes a sonic flow speed of the plasma, since the Mach probe typically measures a sonic ( $M a \square 1$ ) flow speed. The plasma flux estimate almost rises proportional to the applied power. At the maximum power, about $25 \%$ of the injected neutral gas gets ionized.

Figure 9 plots the effect of scanning the injected gas rate for fixed power at $1000 \mathrm{~W}$. Below flow rates of 
about $5 \times 10^{19} / \mathrm{sec}$, high density discharges could not be generated. Above flow rates of about $20 \times 10^{19} / \mathrm{sec}$, the background vacuum chamber neutral pressure is very high and may be effecting the downstream measurements. Between the flow rate limits, the plasma density and flux estimate generally rise with input gas flow rate. The electron temperature trends downward and the profiles tend to broaden. About $25 \%$ of the injected gas becomes ionized, independent of the input flow rate.

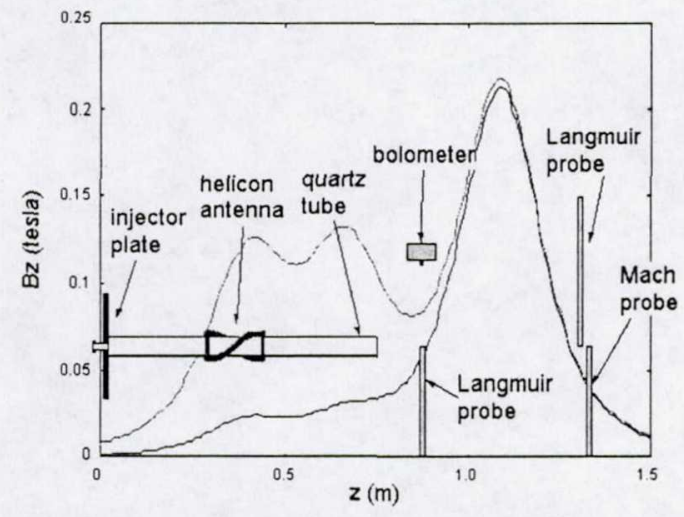

FIG. 10. Two typical magnetic field axial profiles corresponding to Fig. 1b. The helicon antenna configuration and Langmuir probe locations are shown.

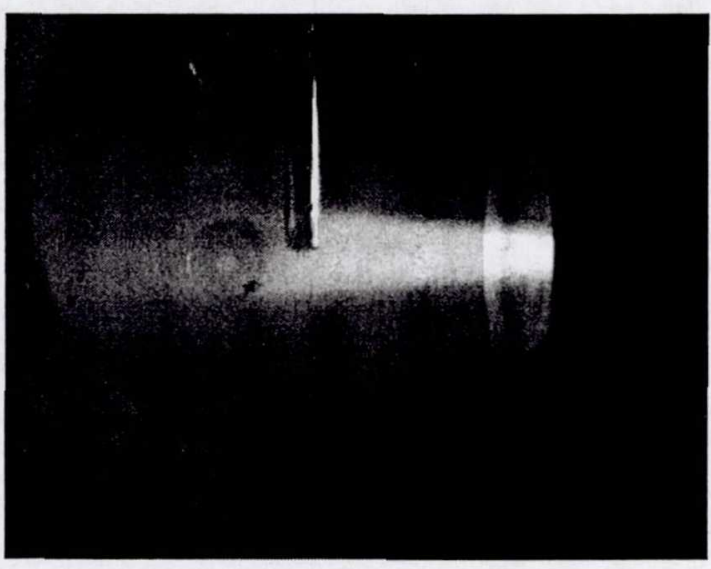

FIG. 11. Hydrogen plasma exhaust with the Langmuir probe inserted.
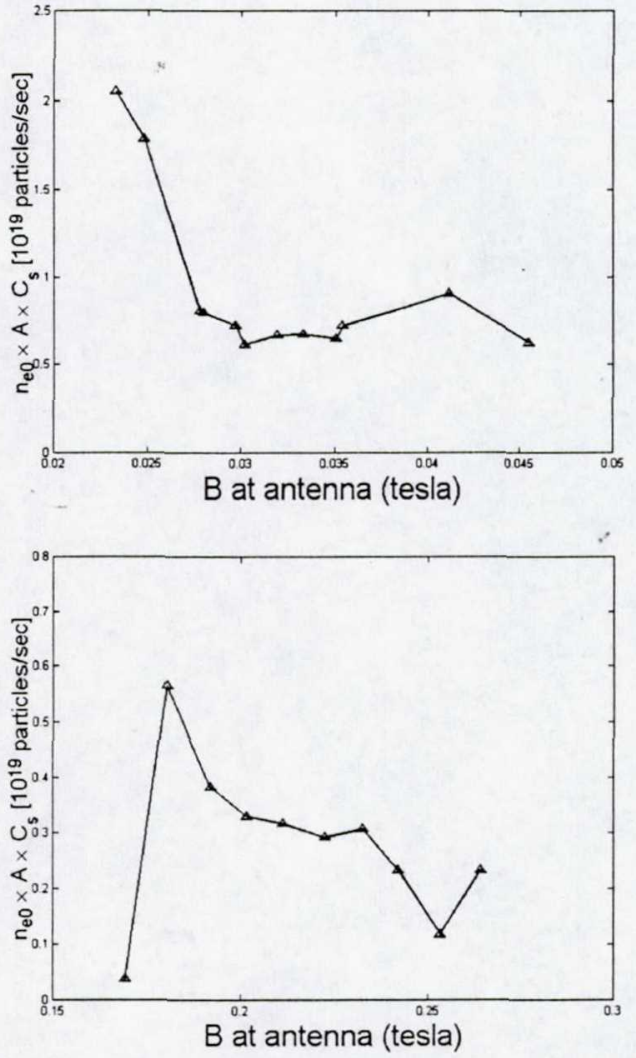

FIG. 12. Plasma flux estimate measure during magnetic field scans for hydrogen discharges with 2000 $\mathrm{W}$ at $25 \mathrm{MHz}$. (a) The field under the helicon antenna scan with the choke field fixed. (b) The choke field scanned with the field at the antenna fixed.

\section{HYDROGEN}

Hydrogen helicon discharge studies performed at the Oak Ridge National Laboratory ${ }^{10}$ discovered that high density plasma production is only achieved near the lower hybrid frequency in the VX-10 type of configuration. We reconfigured the VX-10 device, as figure $1 \mathrm{~b}$ shows, in preparation of hydrogen experiments with a lower hybrid resonance in the helicon section. Figure 10 shows the typical magnetic profile. The plasma exhaust with the Langmuir probe inserted is shown in Fig. 11.

The VX-10 also sees the close connection to the lower hybrid frequency. We see a very strong density dependence on the magnetic field when $\omega_{r f} \approx \omega_{L H}$ as Fig. 12a shows. Here we carefully scanned the magnetic field in the low field region near the antenna. The hydrogen flow was $70 \mathrm{sccm}$ and the input rf power was $2 \mathrm{~kW}$ at $25 \mathrm{MHz}$. We see a strong peak in the density at a specific field strength. This field is somewhat lower than that required for lower hybrid

6 
resonance because the field profile is not flat and the resonance may occur downstream at a higher magnetic field. High density discharges are only achieve when the magnetic polarization is such that the right-hand wave is launched downstream.

The experiments also find that a high magnet field choke is required to achieve high density discharges. Figure $12 b$ shows a sharp cutoff in density at a magnetic choke field of about $0.18 \mathrm{~T}$. This corresponds to a magnetic field ratio from the choke to antenna of over 5. This indicates that some axial confinement may be required.

The hydrogen helicon experiments are fairly recent. Exploration with these type discharges are continuing. We are already seeing nearly $30 \%$ of the incoming gas ionized. The densities are on the order of what is necessary for a plasma thruster device.

\section{FUTURE PLANS}

Intense ICRF studies are beginning. Concomitantly, hydrogen helicon source optimization will continue. Presently, we are installing a high power $(10 \mathrm{~kW})$ vacuum antenna feed for the helicon system. The ICRF system is nearly complete and experiments will begin with this system in June of this year. A substantial helicon source upgrade will be performed by August that will allow nearly steady state high power operation with both the helicon and ICRF. This upgrade will include a larger diameter helicon source and a rf baffle to prevent power deposition of the ICRF on the upstream ion resonance in the source.

\section{CONCLUSION}

The Advanced Space Propulsion Laboratory recently configured a device called VX-10. This device focuses on the development of helicon plasma production and ion cyclotron resonant frequency acceleration to support an early space flight demonstration of the VASIMR concept at a $10 \mathrm{~kW}$ power level. We have described the VX-10 system with several of its diagnostic subsystems. High density helium and hydrogen helicon plasma discharges have been generated. We presented parametric ( $\mathrm{rf}$ power, magnetic field and injected gas flow rate) scans in helium discharges for 3 fixed $\mathrm{rf}$ frequencies $(7,13.56$ and $19 \mathrm{MHz}$ ). The fraction of injected neutral gas that gets ionized increase linearly with rf power and was measured as $25 \%$, at maximum power. The data indicate that higher rf frequency may lead to better efficiency.

Hydrogen discharges with densities in excess of $1 \times 10^{18} / \mathrm{m}^{3}$ were generated with the rf frequency $(25$ $\mathrm{MHz}$ ) near the lower hybrid frequency. Strong dependence on the magnetic field was observed. Also, a downstream magnetic field with a choke ratio of about 5 seems necessary for high density operation. Approximately $30 \%$ of the injected gas was ionized.

Ion cyclotron resonant frequency systems have been installed and tested. Experiments in this area are beginning in the next few months.

\section{NOMENCLATURE}

A Plasma cross sectional area.

$C_{s} \quad$ Plasma sound speed.

$M_{a} \quad$ Mach number.

$m_{i} \quad$ ion mass.

$n_{e 0} \quad$ Central electron density.

$T_{e} \quad$ Electron temperature.

$\mathrm{sccm}$ Standard cubic centimeters per minute.

$\Gamma_{\text {plasma }}$ Plasma flux estimate.

$\omega_{c e}$ electron cyclotron frequency.

$\omega_{c i}$ ion cyclotron frequency.

$\omega_{L H} \quad$ lower hybrid frequency.

$\omega_{p i}$ ion plasma frequency.

$\omega_{r f} \quad$ rf drive frequency.

\section{ACKNOWLEDGMENTS}

This research was sponsored by NASA L. Johnson Space Center. The authors would like to thank Garland (Buddy) Goebel for all of his work putting the experiment together.

\section{REFERENCES}

1. F. F. Chen, Plasma Phys. Controlled Fusion 33, 339 (1991).

2. Porkolab, M., in Fusion, edited by E. Teller, Academic Press, New York (1981), Vol. 1B.

3. F. R. Chang Díaz, et al. "Research Status of the Variable Specific Impulse Magnetoplasma 
Rocket", Transactions of Fusion Technology, 35, 87 (1999).

4. Chang Díaz, this conference.

5. Petro, A. this conference.

6. Schwenterly, S.W., et al, "HTS Magnets for Advanced Magnetoplasma Space Propulsion Applications," to be published in Advances in Cryogenic Engineering, 45 (2000).

7. Chen, S. and Sekiguchi, T., J. Appl. Phys., 36, no. 8, p. 2363.

8. Hutchinson, I.H., Phys. Rev. A, 37, no. 11, p. 4358.

9. Stix, T. Waves in Plasmas (American Institute of Physics, New York, 1992).

10. Goulding, R.H., et al., Bulletin of the American Physical Society, Program of the $41^{\text {st }}$ Annual Meeting of the Division of Plasma Physics, 44, (1999) p99. 